\title{
AN IMPROVED SYMMETRIC SOR ITERATIVE METHOD FOR AUGMENTED SYSTEMS
}

\author{
DAVOD KHOJASTEH SALKUYEH, SOMAYYEH SHAMSI AND AMIR SADEGHI
}

\begin{abstract}
In this paper, the improved symmetric SOR (ISSOR) iterative method is introduced to solve augmented systems. Convergence properties of the proposed method are studied. Some numerical experiments of the ISSOR method are given to compare with that of the well-known SOR-like and MSSOR methods.
\end{abstract}

\section{Introduction}

Consider the augmented system

$$
\left(\begin{array}{cc}
A & B \\
B^{T} & 0
\end{array}\right)\left(\begin{array}{l}
x \\
y
\end{array}\right)=\left(\begin{array}{l}
b \\
q
\end{array}\right),
$$

where $A \in \mathbb{R}^{m \times m}$ is symmetric positive definite, $B \in \mathbb{R}^{m \times n}, m \geq n$ is of column full rank. Obviously, the coefficient matrix of Eq. (1.1) is nonsingular, and hence it has a unique solution. Eq. (1.1) appears in many different applications of scientific computing, such as constrained optimization [12], mixed finite element approximation of elliptic partial differential equations $[4,5,7]$, weighted least-squares problems [3] and others.

The matrices $A$ and $B$ are usually large and sparse. Hence, iterative methods become more attractive than direct methods for solving problem (1.1). There are several iterative methods to solve Eq. (1.1) in the literature. The successive overrelaxation (SOR) [14] is a stationary iterative method which is popular in science and engineering applications. Yuan [15, 16] and Yuan and Iusem [17] have presented several variants of the SOR method and preconditioned conjugate gradient methods to solve general augmented systems such as (1.1) arising from generalized least squares problems where $A$ can be symmetric and positive semidefinite and $B$ can be rank deficient. Golub et al. [8] have presented several SOR-like algorithms to solve augmented systems (1.1). Recently, Darvishi and Hessari [6] applied the symmetric SOR

Corresponding author: Davod Khojasteh Salkuyeh.

2010 Mathematics Subject Classification. 65F10.

Key words and phrases. Augmented system, symmetric positive definite, SOR-like, MSSOR, improved SSOR. 
(SSOR) method to solve (1.1) and then, Wu et al. modified their method (MSSOR) in [13]. Numerical results presented in [13] show that the SOR-like method presented in [8] is superior to the MSSOR method. In this paper we present an improved SSOR (ISSOR) method for solving augmented systems (1.1).

This paper is organized as follows. In section 2, the ISSOR method is presented and its convergence properties are studied. Section 3 is devoted to computing the optimal relaxation parameter of the proposed method. Some numerical experiments are given in section 4 . Section 5 is devoted to the conclusion.

\section{The improved SSOR method}

For the sake of the simplicity, Eq. (1.1) is rewritten in the following form

$$
\left(\begin{array}{cc}
A & B \\
-B^{T} & 0
\end{array}\right)\left(\begin{array}{l}
x \\
y
\end{array}\right)=\left(\begin{array}{c}
b \\
-q
\end{array}\right)
$$

Let

$$
\mathscr{A}=\left(\begin{array}{cc}
A & B \\
-B^{T} & 0
\end{array}\right)=\mathscr{D}-\mathscr{A}_{l}-\mathscr{A}_{u}
$$

where

$$
\mathscr{D}=\left(\begin{array}{ll}
A & 0 \\
0 & Q
\end{array}\right), \quad \mathscr{A}_{l}=\left(\begin{array}{cc}
-\frac{1}{2} A & 0 \\
B^{T} & \frac{1}{2} Q
\end{array}\right), \quad \mathscr{A}_{u}=\left(\begin{array}{cc}
\frac{1}{2} A & -B \\
0 & \frac{1}{2} Q
\end{array}\right),
$$

in which $Q \in \mathbb{R}^{n \times n}$ is a nonsingular symmetric matrix. We set

$$
\mathscr{L}=\mathscr{D}^{-1} \mathscr{A}_{l}=\left(\begin{array}{cc}
-\frac{1}{2} I & 0 \\
Q^{-1} B^{T} & \frac{1}{2} I
\end{array}\right), \quad \mathscr{U}=\mathscr{D}^{-1} \mathscr{A}_{u}=\left(\begin{array}{cc}
\frac{1}{2} I & -A^{-1} B \\
0 & \frac{1}{2} I
\end{array}\right),
$$

where $I$ is the identity matrix. Let

$$
z^{(k)}=\left(\begin{array}{c}
x^{(k)} \\
y^{(k)}
\end{array}\right), \quad \mathscr{C}=\left(\begin{array}{c}
b \\
-q
\end{array}\right)
$$

where $z^{(k)}$ is the $k$ th approximation of solution Eq. (2.1) by the SSOR method using splitting (2.2). In this case, by the forward SOR method we have

$$
z^{\left(k+\frac{1}{2}\right)}=\mathscr{L}_{\omega} z^{(k)}+\omega(I-\omega \mathscr{L})^{-1} \mathscr{D}^{-1} \mathscr{C},
$$

where

$$
\mathscr{L}_{\omega}=(I-\omega \mathscr{L})^{-1}[(1-\omega) I+\omega \mathscr{U}]=\left(\begin{array}{cc}
\frac{2-\omega}{2+\omega} I & -\frac{2 \omega}{2+\omega} A^{-1} B \\
\frac{2 \omega}{2+\omega} Q^{-1} B^{T} & I-\frac{4 \omega^{2}}{4-\omega^{2}} Q^{-1} B^{T} A^{-1} B
\end{array}\right) .
$$


Note that

$$
I-\omega \mathscr{L}=\left(\begin{array}{cc}
\left(1+\frac{1}{2} \omega\right) I & 0 \\
-\omega Q^{-1} B^{T} & \left(1-\frac{1}{2} \omega\right) I
\end{array}\right)
$$

Therefore

$$
\operatorname{det}(I-\omega \mathscr{L})=\left(1+\frac{1}{2} \omega\right)^{m}\left(1-\frac{1}{2} \omega\right)^{n} \neq 0,
$$

if and only if $\omega \neq \pm 2$.

Also, by the backward SOR method, from $z^{\left(k+\frac{1}{2}\right)}$, we compute $z^{(k+1)}$ via

$$
z^{(k+1)}=\mathscr{U}_{\omega} z^{\left(k+\frac{1}{2}\right)}+\omega(I-\omega \mathscr{U})^{-1} \mathscr{D}^{-1} \mathscr{C},
$$

where

$$
\mathscr{U}_{\omega}=(I-\omega \mathscr{U})^{-1}[(1-\omega) I+\omega \mathscr{L}]=\left(\begin{array}{cc}
\frac{2-3 \omega}{2-\omega} I-\frac{4 \omega^{2}}{(2-\omega)^{2}} A^{-1} B Q^{-1} B^{T} & -\frac{2 \omega}{(2-\omega)} A^{-1} B \\
\frac{2 \omega}{2-\omega} Q^{-1} B^{T} & I
\end{array}\right) .
$$

Here, we mention that

$$
I-\omega \mathscr{U}=\left(\begin{array}{cc}
\left(1-\frac{1}{2} \omega\right) I & \omega A^{-1} B \\
0 & \left(1-\frac{1}{2} \omega\right) I
\end{array}\right)
$$

Hence

$$
\operatorname{det}(I-\omega \mathscr{U})=\left(1-\frac{1}{2} \omega\right)^{m+n} \neq 0,
$$

if and only if $\omega \neq 2$.

Now, assuming that $\omega \neq \pm 2$, from Eqs. (2.3) and (2.4) we obtain the ISSOR method as following

$$
z^{(n+1)}=\mathscr{J}_{\omega} z^{(n)}+\mathscr{K}
$$

where

$$
\mathscr{J}_{\omega}=\mathscr{U}_{\omega} \mathscr{L}_{\omega}=\left(\begin{array}{cc}
\frac{2-3 \omega}{2+\omega} I-\frac{8 \omega^{2}}{4-\omega^{2}} A^{-1} B Q^{-1} B^{T} & -\frac{4 \omega}{2+\omega} A^{-1} B+\frac{16 \omega^{2}}{(2-\omega)\left(4-\omega^{2}\right)} A^{-1} B Q^{-1} B^{T} A^{-1} B \\
\frac{4 \omega}{2+\omega} Q^{-1} B^{T} & I-\frac{8 \omega^{2}}{4-\omega^{2}} Q^{-1} B^{T} A^{-1} B
\end{array}\right),
$$

and

$$
\begin{aligned}
\mathscr{K} & =\omega(2-\omega)(I-\omega \mathscr{U})^{-1}(I-\omega \mathscr{L})^{-1} \mathscr{D}^{-1} \mathscr{C} \\
& =\left(\begin{array}{c}
\frac{4 \omega}{2+\omega} A^{-1} b-\frac{16 \omega^{2}}{(2-\omega)\left(4-\omega^{2}\right)} A^{-1} B Q^{-1} B^{T} A^{-1} b+\frac{8 \omega^{2}}{(2-\omega)^{2}} A^{-1} B Q^{-1} q \\
\frac{8 \omega^{2}}{4-\omega^{2}} Q^{-1} B^{T} A^{-1} b-\frac{4 \omega}{2-\omega} Q^{-1} q
\end{array}\right) .
\end{aligned}
$$

We also have

$$
\begin{aligned}
\mathscr{J}_{\omega} & =\mathscr{U}_{\omega} \mathscr{L}_{\omega}=(I-\omega \mathscr{U})^{-1}[(1-\omega) I+\omega \mathscr{L}](I-\omega \mathscr{L})^{-1}[(1-\omega) I+\omega \mathscr{U}] \\
& =I-\omega(2-\omega)(I-\omega \mathscr{U})^{-1}(I-\omega \mathscr{L})^{-1} \mathscr{D}^{-1} \mathscr{A}
\end{aligned}
$$




$$
=[(I-\omega \mathscr{L})(I-\omega \mathscr{U})]^{-1}\left[(I-\omega \mathscr{L})(I-\omega \mathscr{U})-\omega(2-\omega) \mathscr{D}^{-1} \mathscr{A}\right] .
$$

By some simple manipulations, from the recurrence (2.5), we can summarize the ISSOR method as the Algorithm 1.

\section{Algorithm 1: The ISSOR method}

1. Choose the nonsingular symmetric matrix $Q$ and initial guesses $x^{(0)}$ and $y^{(0)}$, and a relaxation parameter $\omega>0$.

2. For $k=0,1,2, \ldots$, until convergence Do,

$$
\begin{aligned}
y^{(k+1)} & =y^{(k)}+\frac{4 \omega}{2+\omega} Q^{-1} B^{T}\left\{x^{(k)}+\frac{2 \omega}{2-\omega} A^{-1}\left(b-B y^{(k)}\right)\right\}-\frac{4 \omega}{2-\omega} Q^{-1} q, \\
x^{(k+1)} & =\frac{2-3 \omega}{2+\omega} x^{(k)}-\frac{2 \omega}{2-\omega} A^{-1} B\left\{y^{(k+1)}+\frac{2-3 \omega}{2+\omega} y^{(k)}\right\}+\frac{4 \omega}{2+\omega} A^{-1} b .
\end{aligned}
$$

3. EndDo

Here, it is necessary to mention that, in this algorithm, the matrix $Q$ is an approximation of the Schur complement matrix $B^{T} A^{-1} B$ (for more details see [8] ). A comparison between the ISSOR and the MSSOR methods show that only the constant coefficients of these algorithms are different.

To study the convergence properties of the proposed method we state and prove the following theorem.

Theorem 1. Suppose that $\mu$ is an eigenvalue of $Q^{-1} B^{T} A^{-1} B$. If $\lambda$ satisfies

$$
(\lambda-1)(2-\omega)((2-3 \omega)-(2+\omega) \lambda)=16 \omega^{2} \lambda \mu,
$$

then $\lambda$ is an eigenvalue of $\mathscr{J}_{\omega}$. Conversely, if $\lambda$ is an eigenvalue of $\mathscr{J}_{\omega}$ such that $\lambda \neq \frac{2-3 \omega}{2+\omega}, \lambda \neq 1$ and $\mu$ satisfies (2.7), then $\mu$ is a nonzero eigenvalue of $Q^{-1} B^{T} A^{-1} B$.

Proof. Let $(\lambda, x)$ be an eigenpair of $\mathscr{J}_{\omega}$, i.e.,

$$
\mathscr{J}_{\omega} x=\lambda x
$$

Therefore, from (2.6) we obtain

$$
[(I-\omega \mathscr{L})(I-\omega \mathscr{U})]^{-1}\left[(I-\omega \mathscr{L})(I-\omega \mathscr{U})-\omega(2-\omega) \mathscr{D}^{-1} \mathscr{A}\right] x=\lambda x,
$$

or

$$
\left[(I-\omega \mathscr{L})(I-\omega \mathscr{U})-\omega(2-\omega) \mathscr{D}^{-1} \mathscr{A}\right] x=\lambda[(I-\omega \mathscr{L})(I-\omega \mathscr{U})] x
$$

which is equivalent to

$$
(1-\lambda)(I-\omega \mathscr{L})(I-\omega \mathscr{U}) x=\omega(2-\omega) \mathscr{D}^{-1} \mathscr{A} x .
$$


Therefore

$(1-\lambda)\left(\begin{array}{cc}\left(1-\frac{1}{4} \omega^{2}\right) I & \omega\left(1+\frac{1}{2} \omega\right) A^{-1} B \\ -\omega\left(1-\frac{1}{2} \omega\right) Q^{-1} B^{T} & \left(1-\frac{1}{2} \omega\right)^{2} I-\omega^{2} Q^{-1} B^{T} A^{-1} B\end{array}\right)\left(\begin{array}{l}x_{1} \\ x_{2}\end{array}\right)=\omega(2-\omega)\left(\begin{array}{cc}I & A^{-1} B \\ -Q^{-1} B^{T} & 0\end{array}\right)\left(\begin{array}{l}x_{1} \\ x_{2}\end{array}\right)$.

From this we obtain the following system of two equations

$$
\left\{\begin{array}{l}
\left((1-\lambda)\left(1-\frac{1}{4} \omega^{2}\right)-\omega(2-\omega)\right) x_{1}=\left(\omega(2-\omega)-(1-\lambda) \omega\left(1+\frac{1}{2} \omega\right)\right) A^{-1} B x_{2}, \\
\omega^{2}(1-\lambda) Q^{-1} B^{T} A^{-1} B x_{2}-(1-\lambda)\left(1-\frac{1}{2} \omega\right)^{2} x_{2}=\omega(2-\omega)\left(1-\frac{1}{2}(1-\lambda)\right) Q^{-1} B^{T} x_{1},
\end{array}\right.
$$

From the the first equation, we have

$$
x_{1}=\frac{2 \omega(2-3 \omega+\lambda(2+\omega))}{(2-\omega)(2-3 \omega-\lambda(2+\omega))} A^{-1} B x_{2},
$$

and substituting $x_{1}$ into the second equation, yields

$$
(1-\lambda)\left(1-\frac{1}{2} \omega\right)^{2} x_{2}-\omega^{2}(1-\lambda) Q^{-1} B^{T} A^{-1} B x_{2}=-\frac{\omega^{2}(2-3 \omega+\lambda(2+\omega))(1+\lambda)}{(2-3 \omega-\lambda(2+\omega))} Q^{-1} B^{T} A^{-1} B x_{2} .
$$

Therefore,

$$
\begin{aligned}
& (1-\lambda)\left(1-\frac{1}{2} \omega\right)^{2}(2-3 \omega-\lambda(2+\omega)) x_{2} \\
& \quad=\left[\omega^{2}(1-\lambda)(2-3 \omega-\lambda(2+\omega))-\omega^{2}(2-3 \omega+\lambda(2+\omega))(1+\lambda)\right] Q^{-1} B^{T} A^{-1} B x_{2} .
\end{aligned}
$$

Now, suppose that $\mu$ is an eigenvalue of $Q^{-1} B^{T} A^{-1} B$. Then we have

$$
(\lambda-1)(2-\omega)^{2}((2-3 \omega-\lambda(2+\omega)))=16 \omega^{2} \lambda(2-\omega) \mu .
$$

Hence

$$
(\lambda-1)(2-\omega)(2-3 \omega-\lambda(2+\omega))=16 \omega^{2} \lambda \mu .
$$

We can prove the second assertion by reversing the process.

Lemma 1. Consider the quadratic equation $x^{2}-b x+c=0$, where $b$ and $c$ are real numbers. Both roots of the equation are less than one in modulus if and only if $|c|<1$ and $|b|<1+c$.

Proof. See Young [14].

Theorem 2. Suppose that $B$ has full rank and $A$ is symmetric and positive definite. Assume that all eigenvalues $\mu$ of $Q^{-1} B^{T} A^{-1} B$ are real and positive. Then, the ISSOR method is convergent if

$$
0<\omega<\frac{2}{1+2 \sqrt{\rho}}
$$

where $\rho$ is the spectral radius of $Q^{-1} B^{T} A^{-1} B$. 
Proof. After some manipulations on Eq. (2.7), we get

$$
\lambda^{2}-2 \frac{(2-\omega)^{2}-8 \omega^{2} \mu}{4-\omega^{2}} \lambda+\frac{2-3 \omega}{2+\omega}=0 .
$$

Let

$$
b=-2 \frac{(2-\omega)^{2}-8 \omega^{2} \mu}{4-\omega^{2}}, \quad c=\frac{2-3 \omega}{2+\omega} .
$$

Now, from Lemma $1,|\lambda|<1$ if and only if

$$
\left|\frac{2-3 \omega}{2+\omega}\right|<1
$$

and

$$
\left|-2 \frac{(2-\omega)^{2}-8 \omega^{2} \mu}{4-\omega^{2}}\right|<1+\frac{2-3 \omega}{2+\omega} .
$$

It is easy to see that inequality (2.9) is equivalent to

$$
0<\omega<2 \text {. }
$$

On the other hand, inequality (2.10) may be rewritten in the form

$$
-\frac{2-\omega}{2+\omega}<\frac{(2-\omega)^{2}-8 \omega^{2} \mu}{(2-\omega)(2+\omega)}<\frac{2-\omega}{2+\omega},
$$

which is itself equivalent to

$$
-2(2-\omega)^{2}<-8 \omega^{2} \mu<0 .
$$

Since $\mu>0$ and $0<\omega<2$, the right inequality in (2.11) is always true. On the other hand, the left inequality can be written as

$$
[2 \omega \sqrt{\mu}-(2-\omega)][2 \omega \sqrt{\mu}+(2-\omega)]<0 .
$$

Since $2 \omega \sqrt{\mu}+(2-\omega)>0$, the latter inequality is true if and only if

$$
2 \omega \sqrt{\mu}-(2-\omega)<0,
$$

or

$$
0<\omega<\frac{2}{1+2 \sqrt{\mu}} .
$$

Since $\mu>0$, we have $2 /(1+2 \sqrt{\mu})<2$. Therefore we conclude that $|\lambda|<1$ if and only if

$$
0<\omega<\frac{2}{1+2 \sqrt{\mu}}
$$

and this completes the proof.

\section{Determination of the optimum relaxation parameter}

In this section, we present the following theorem which gives the optimum relaxation parameter of the ISSOR method. For the sake of the simplicity, let $\rho=\rho\left(Q^{-1} B^{T} A^{-1} B\right)$ and $0<\mu_{0}=\min _{\mu \neq 0} \mu$, where $\mu$ is a nonzero eigenvalue of $Q^{-1} B^{T} A^{-1} B$. 
Theorem 3. If $\mu_{0} \geq \frac{1}{2}$, then

$$
\rho\left(\mathscr{J}_{\omega}\right)= \begin{cases}\sqrt{\frac{2-3 \omega}{2+\omega}}, & \text { if } 0<\omega \leq \frac{2 \sqrt{4 \rho-1}}{4 \rho+\sqrt{4 \rho-1}}, \\ \frac{1}{4-\omega^{2}}\left[\left|(2-\omega)^{2}-8 \omega^{2} \rho\right|+2 \omega \sqrt{(2-\omega)^{2}(1-4 \rho)+16 \omega^{2} \rho^{2}}\right], & \text { if } \frac{2 \sqrt{4 \rho-1}}{4 \rho+\sqrt{4 \rho-1}} \leq \omega<\frac{2}{1+2 \sqrt{\rho}} .\end{cases}
$$

Moreover, the optimal parameter $\omega_{\text {opt }}$ and $\rho\left(\mathscr{J}_{\text {opt }}\right)$ are given by

$$
\omega_{\text {opt }}=\frac{2 \sqrt{4 \rho-1}}{4 \rho+\sqrt{4 \rho-1}} \quad \text { and } \quad \rho\left(\mathscr{J}_{\text {opt }}\right)=\sqrt{\frac{2 \rho-\sqrt{4 \rho-1}}{2 \rho+\sqrt{4 \rho-1}}} .
$$

Proof. From the quadratic equation (2.8), we obtain

$$
\lambda=\frac{1}{4-\omega^{2}}\left[(2-\omega)^{2}-8 \omega^{2} \mu \pm 2 \omega \sqrt{\delta}\right]
$$

where

$$
\begin{aligned}
\delta & =(2-\omega)^{2}(1-4 \mu)+16 \omega^{2} \mu^{2}=16 \omega^{2} \mu^{2}-(2-\omega)^{2}(4 \mu-1) \\
& =(4 \omega \mu-(2-\omega) \sqrt{4 \mu-1})(4 \omega \mu+(2-\omega) \sqrt{4 \mu-1}) .
\end{aligned}
$$

Since $4 \omega \mu+(2-\omega) \sqrt{4 \mu-1}>0$, we conclude that $\lambda$ is complex if

$$
4 \omega \mu-(2-\omega) \sqrt{4 \mu-1}<0,
$$

which is equivalent to

$$
\omega<f(\mu)=\frac{2 \sqrt{4 \mu-1}}{4 \mu+\sqrt{4 \mu-1}} .
$$

Obviously $f(\mu)<2$. Therefore, if $0<\omega<f(\mu)$, then $\delta<0$, and if $f(\mu) \leq \omega<2$, then $\delta \geq 0$. Hence, we have

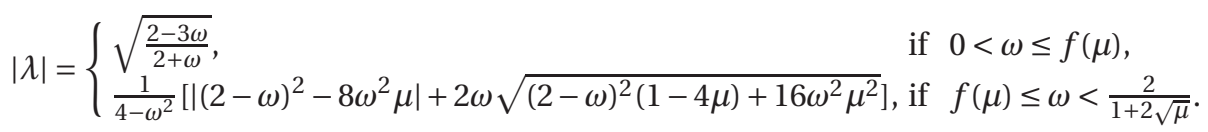

It is easy to see that for all $\mu \geq \frac{1}{2}$, the function $f(\mu)$ is monotonically decreasing function. Therefore, we get

$$
\rho\left(\mathscr{J}_{\omega}\right)= \begin{cases}\sqrt{\frac{2-3 \omega}{2+\omega}}, & \text { if } 0<\omega \leq f(\rho), \\ \frac{1}{4-\omega^{2}}\left[\left|(2-\omega)^{2}-8 \omega^{2} \rho\right|+2 \omega \sqrt{(2-\omega)^{2}(1-4 \rho)+16 \omega^{2} \rho^{2}}\right], & \text { if } f(\rho) \leq \omega<\frac{2}{1+2 \sqrt{\rho}} .\end{cases}
$$

To compute the optimal relaxation parameter, we rewrite (2.8) in the form

$$
f_{\omega}(\lambda)=g_{\omega}(\lambda)
$$

where

$$
f_{\omega}(\lambda)=\frac{4-\omega^{2}}{\omega^{2}}\left(\lambda-\frac{2-\omega}{2+\omega}\right)^{2}-\frac{4(2-\omega)}{2+\omega}
$$


Table 1: Choices of matrix $Q$.

\begin{tabular}{lll}
\hline Case no. & Matrix $Q$ & Description \\
\hline I & $B^{T} \hat{A}^{-1} B$ & $\hat{A}=\operatorname{tridiag}(A)$ \\
II & $B^{T} \hat{A}^{-1} B$ & $\hat{A}=\operatorname{diag}(A)$ \\
\hline
\end{tabular}

$$
g_{\omega}(\lambda)=-16 \mu \lambda
$$

It is easy to see that $f_{\omega}$ and $g_{\omega}$ pass through $(1,0)$ and $(0,0)$, respectively, i.e., $f_{\omega}(1)=g_{\omega}(0)=0$. The straight line $g_{\omega}$ crosses the parabolic curve $f_{\omega}$. Analogous to analysis in [11, pp. 110-111], the optimal relaxation parameter $\omega_{\text {opt }}$ is the choice that guarantees that $f_{\omega_{o p t}}$ is tangent line of $g_{\omega_{\text {opt }}}$. Using the same idea, we get

$$
\omega_{o p t}=\frac{2 \sqrt{4 \rho-1}}{4 \rho+\sqrt{4 \rho-1}} \quad \text { and } \quad \rho\left(\mathscr{J}_{\text {opt }}\right)=\sqrt{\frac{2 \rho-\sqrt{4 \rho-1}}{2 \rho+\sqrt{4 \rho-1}}} .
$$

\section{Numerical experiments}

In this section, we present some numerical experiments to compare the ISSOR method with the SOR-like and MSSOR methods. All the numerical results presented in this section were computed by some MATLAB codes in double precision. The initial guess was always $\left(x^{(0)^{T}}, y^{(0)^{T}}\right)^{T}=0$ and the right hand side $\left(b^{T}, q^{T}\right)^{T}$ was selected such that the exact solution of the augmented system $(1.1)$ is $\left(x^{(*)^{T}}, y^{(*)^{T}}\right)^{T}=(1,1, \ldots, 1)^{T}$. The stopping criterion

$$
\operatorname{Err}_{k}=\frac{\sqrt{\left\|x^{(k)}-x^{(*)}\right\|_{2}^{2}+\left\|y^{(k)}-y^{(*)}\right\|_{2}^{2}}}{\sqrt{\left\|x^{(0)}-x^{(*)}\right\|_{2}^{2}+\left\|y^{(0)}-y^{(*)}\right\|_{2}^{2}}}<10^{-12},
$$

was used in the computations. The preconditioning matrix $Q$, which is an approximation of $B^{T} A^{-1} B$, is chosen according to the cases listed in Table 1 .

Example 1. Consider the augmented linear system (1.1) with [13]

$$
A=\left(\begin{array}{cc}
I \otimes T+T \otimes I & 0 \\
0 & I \otimes T+T \otimes I
\end{array}\right) \in \mathbb{R}^{2 p^{2} \times 2 p^{2}}, \quad B=\left(\begin{array}{c}
I \otimes F \\
F \otimes I
\end{array}\right) \in \mathbb{R}^{2 p^{2} \times p^{2}},
$$

and

$$
T=\frac{1}{h^{2}} \operatorname{tridiag}(-1,2,-1) \in \mathbb{R}^{p \times p}, \quad F=\frac{1}{h} \operatorname{tridiag}(-1,1,0) \in \mathbb{R}^{p \times p},
$$

where $\otimes$ is the Kronecker product symbol and $h=\frac{1}{p+1}$ is the discretization mesh-size. Let $m=2 p^{2}$ and $n=p^{2}$. Hence, the total number of variables is $m+n=3 p^{2}$. In Table 2 , the 
Table 2: Numerical results for Example 1.

\begin{tabular}{lllll}
\hline Method & & ISSOR & MSSOR & SOR-like \\
\hline \hline Case I $\left(\mu_{0}=0.5319\right)$ & $\omega_{\text {opt }}$ & 0.3037 & 0.3081 & 0.5958 \\
& $\rho(\cdot)$ & 0.6875 & 0.6919 & 0.6358 \\
& Its & 96 & 98 & 78 \\
\hline Case II $\left(\mu_{0}=0.5162\right)$ & $\omega_{\text {opt }}$ & 0.2356 & 0.2375 & 0.4664 \\
& $\rho(\cdot)$ & 0.7606 & 0.7625 & 0.7305 \\
& Its & 134 & 135 & 114 \\
\hline
\end{tabular}

minimum positive eigenvalue of $Q^{-1} B^{T} A^{-1} B, \mu_{0}$, for $p=8(m=128$ and $n=64)$ is given. Numerical results of the SOR-like, MSSOR and ISSOR methods are given in Table 2 . In Table 2, the optimum relaxation parameter $w_{\text {opt }}$ together with its corresponding spectral radius denoted by $\rho(\cdot)$ are given. In this table, "Its" stands for the number of iterations for the convergence. As we observe for the both cases we have $\mu_{0}>0.5$. Hence, we can use Theorem 3 for computing the optimal relaxation parameter $\omega_{\text {opt }}$. Here we mention that we used Theorem 4 in [13] and Theorem 3.1 in [8] for computing the optimal relaxation parameters of the MSSOR and SOR-like methods, respectively. Numerical results presented in Table 2 show that the ISSOR method is slightly better than the MSSOR method, and the SOR-like method is better than the other two methods. Convergence curves $\left(\log _{10} \operatorname{Err}_{k}\right)$ of the methods are depicted in Figure 1 .

Example 2. In this example, we consider the augmented linear system (1.1) with $A=U^{T} U$, where $U=\left(u_{i j}\right) \in \mathbb{R}^{2 p^{2} \times 2 p^{2}}$ is an upper triangular matrix with entries

$$
u_{i j}=\left\{\begin{array}{l}
1, i=j \\
\alpha, i<j \\
0, i>j
\end{array}\right.
$$

in which $\alpha \in \mathbb{R}$. Obviously $A$ is a symmetric positive definite matrix. $A$ is called a Moler matrix and one may use the MATLAB command

$$
\mathrm{A}=\operatorname{gallery}(\text { 'moler', } \mathrm{n}, \mathrm{al} \mathrm{pha}) \text {, }
$$

to produce a Moler matrix of dimension $n$. We also consider the matrix $B=\left(b_{i j}\right) \in \mathbb{R}^{2 p^{2} \times p^{2}}$ with

$$
b_{i j}=\left\{\begin{array}{l}
j, i=j+m-n, \\
0, \text { otherwise. }
\end{array}\right.
$$

We assume that $p=12$ and $\alpha=0.005$. In this case, $\mathscr{A}$ is of order $432 \times 432$. All of the assumptions and notations are as before. Numerical results are given in Table 3 and the convergence curves of the methods are depicted in Figure 2. Table 3 and Figure 2 show that the ISSOR method is superior to the MSSOR and SOR-like methods. An important observation can be 

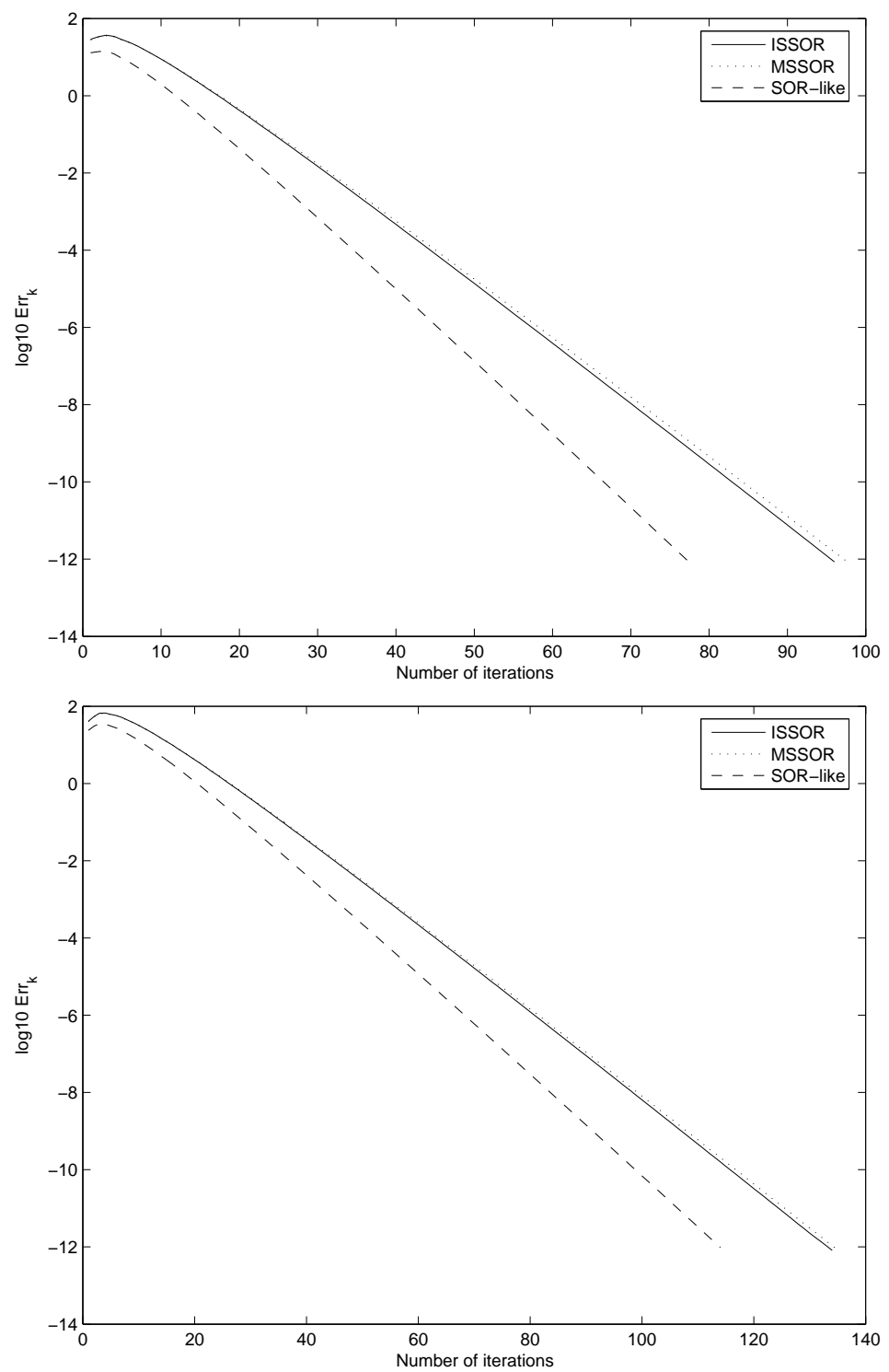

Figure 1: Convergence history of the methods for Example 1; case 1 (up), case 2 (down).

posed here. We see that the spectral radius of the SOR-like method is smaller than that of the other two methods. Nevertheless, the number of iterations is greater that of the other two methods. We believe that a catastrophic cancelation [18] has been occurred here. Since, in both cases the optimal relaxation parameter is almost equal to 1 . On the other hand, by referring to the SOR-like algorithm (see [8, page 73]), we observe that $1-\omega$ is used in the recurrence of this algorithm. 

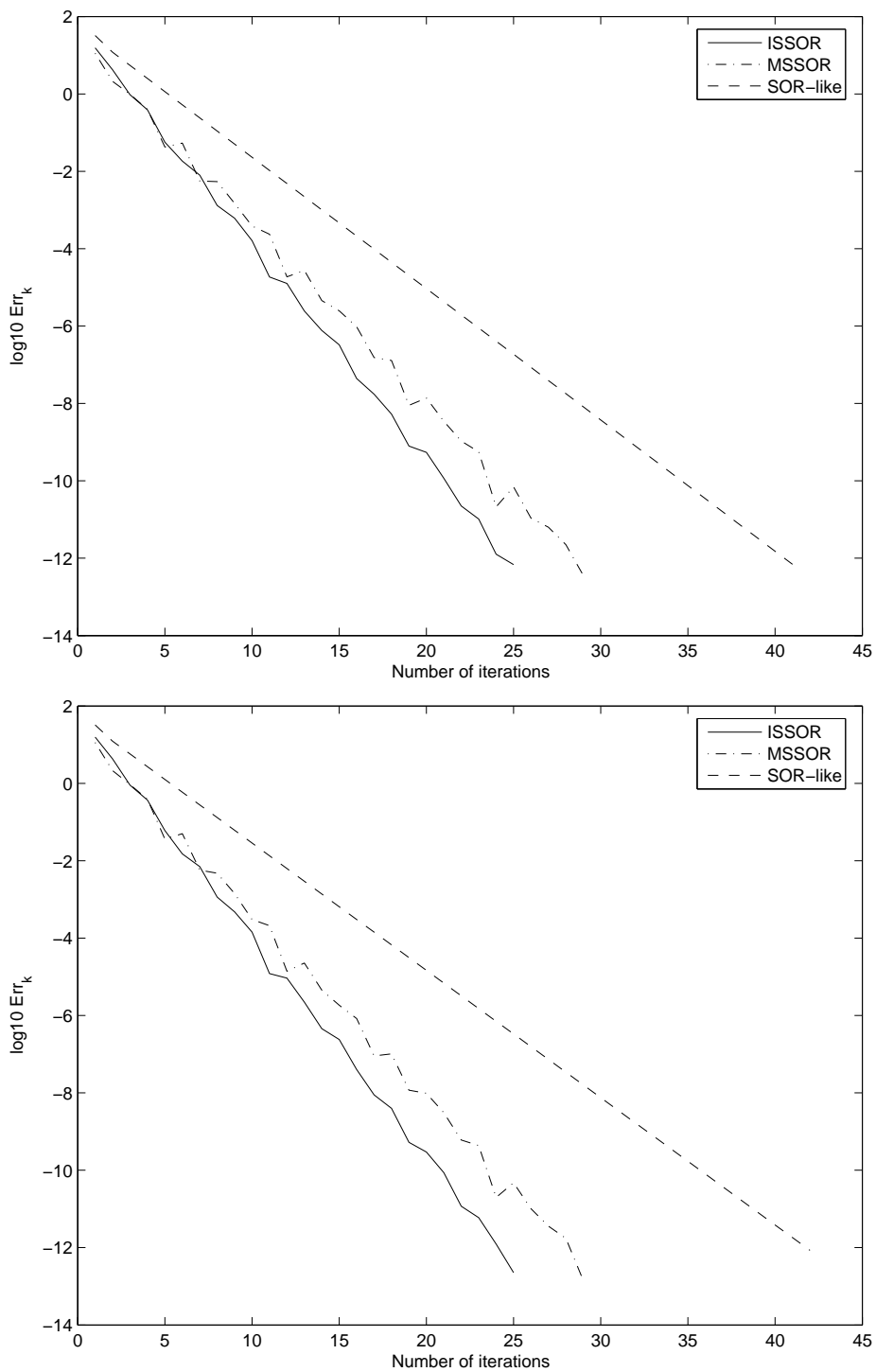

Figure 2: Convergence history of the methods for Example 2; case 1 (up), case 2 (down).

\section{Conclusion}

In this paper, we have presented the ISSOR iterative method to solve augmented linear system of equations. This method may be considered and an improved version of the SSOR method presented by Darvishi and Hessari in [6]. Numerical results show that the proposed method is superior to the MSSOR method presented by Wu et al. in [13], but in the current form, it can not compared with the SOR-like method. As it has been mentioned in [13], further improvement of the ISSOR method can be done similar to the idea of [2]. This will be studied in future. 
Table 3: Numerical results for Example 2.

\begin{tabular}{lllll}
\hline Method & & ISSOR & MSSOR & SOR-like \\
\hline \hline Case I $\left(\mu_{0}=0.5423\right)$ & $\omega_{\text {opt }}$ & 0.5996 & 0.6592 & 0.9997 \\
& $\rho(\cdot)$ & 0.2783 & 0.3408 & 0.0167 \\
& Its & 25 & 29 & 41 \\
\hline Case II $\left(\mu_{0}=0.5312\right)$ & $\omega_{\text {opt }}$ & 0.6026 & 0.6640 & 1.0000 \\
& $\rho(\cdot)$ & 0.2717 & 0.3360 & 0.0061 \\
& Its & 25 & 29 & 42 \\
\hline
\end{tabular}

\section{References}

[1] O. Axelsson, Iterative solution method, Cambridge University Press, Cambridge, 1996.

[2] Z. Z. Bai, B. N. Parlett and Z. Q. Wang, On generalized successive overrelaxtion methods for augmented systems, Numer. Math. 102 (2005), 1-38

[3] A. Bjorck, Numerical methods for least squares problems, SIAM, Philadelphia, PA, 1996.

[4] S. C. Brenner and L. R. Scott, The mathematical theory of finite element methods, Second edition, SpringerVerlag, New York ,2002.

[5] F. Brezzi and M. Fortin, Mixed and hybrid finite element methods, Springer-Verlag, New York, 1991.

[6] M.T. Darvishi and P. Hessari, Symmetric SOR method for augmented systems, Appl. Math. Comput. 183, (2006) 409-415.

[7] H. Elman and D. Silvester, Fast nonsymmetric iteration and preconditioning for Navier- Stokes equations, SIAM J. Sci. Comput. 17 (1996), 33-46.

[8] G.H. Golub, X. Wu, J.Y. Yuan, SOR-like methods for augmented systems, BIT 41 (2001), 71-85.

[9] A. Hadjidimos, Accelerated overrelaxation method, Math. Comput. 32 (1978), 149-157.

[10] Y. Saad, Iterative Methods for Sparse linear Systems, PWS press, New York, 1995.

[11] R. S. Varga, Matrix iterative analysis, Prentice-Hall, Englewood Cliffs, NJ, 1962.

[12] S. Wright, Stability of augmented system factorizations in interior-point methods, SIAM J. Matrix Anal. Appl. 18 (1997), 191-222.

[13] S. L. Wu, T.Z. Huang and X.L. Zhao, A modified SSOR iterative method for augmented systems, J. Comput. Appl. Math. 228 (2009), 424-433.

[14] D.M. Young, Iterative Solution for Large Linear Systems, Academic Press, New York, 1971.

[15] J.Y. Yuan, Iterative methods for generalized least squares problems, Ph.D. Thesis, IMPA, Rio de Janeiro, Brazil, 1993.

[16] J.Y. Yuan, Numerical methods for generalized least squares problems, J. Comput. Appl. Math. 66 (1996), 571584.

[17] J.Y. Yuan, A.N. Iusem, Preconditioned conjugate gradient methods for generalized least squares problems, J. Comput. Appl. Math. 71 (1996), 287-297.

[18] C. J. Zarowski, An introduction to numerical analysis for electrical and computer engineers, John Wiley \& Sons, Hoboken, New Jersey, 2004.

Faculty of Mathematical Sciences, University of Guilan, P.O. Box 1914, Rasht, Iran.

E-mail: khojasteh@guialn.ac.ir

Young Researchers Club, Ardabil branch, Islamic Azad University, Ardabil, Iran.

E-mail: salkuyeh@gmail.com

Young Researchers Club, Ardabil branch, Islamic Azad University, Ardabil, Iran.

E-mail: amir_math20@yahoo.com 